\title{
DESARROLLO PRÁCTICO DE LAS \\ NOTIFICACIONES JUDICIALES MEDIANTE SISTEMAS ELECTRÓNICOS*
}

\section{Practical development of the judgment notification through electronic systems}

Sebastián Portilla Parra**

\section{Resumen:}

Las notificaciones electrónicas tienen como finalidad agilizar el proceso, y para ser eficaces deben cumplir con la integralidad e inalterabilidad de su contenido, pues representan una nueva posibilidad en la función jurisdiccional del Estado. Aquellas, deberían tener aplicación en cualquier tipo de proceso judicial, sin embargo no han logrado posicionarse como un tipo de notificación principal, pues su carácter aún es facultativo y subsidiario. En el presente trabajo se analizará su desarrollo en los procesos judiciales, enfatizando en su evolución dentro del proceso administrativo y verbal. De igual forma, a la posterior recolección, análisis e interpretación de doctrina, legislación, jurisprudencia y derecho comparado correspondiente al tema, tendremos un compendio de características propias como, principios determinados, posibles mecanismos de seguridad y legislación especial. Aunque preocupa la seguridad brindada para su uso, por la poca diligencia del Estado y la necesidad de un sistema seguro para implementarlas a los diferentes procesos.

Recebido: 28 de octubre de 2016 - Aprobado: 30 de abril de 2017

Artículo Inédito.

Para citar el artículo: PORTILLA PARRA, Sebastián. Desarrollo práctico de las notificaciones judiciales mediante sistemas electrónicos. Revista del Instituto Colombiano de Derecho Procesal. No. 45 Enero - Junio. 2017, pp. 189-213.

** Estudiante de la Facultad de Derecho, cursa VII semestre, Universidad del Cauca. 
Palabras clave: Notificaciones electrónicas, implementación, derechos fundamentales, proceso verbal, proceso administrativo.

\begin{abstract}
Electronic notifications are intended to streamline the process, and to be effective they must comply with the integrality and inalterability of their content, since they represent a new possibility in the jurisdictional function of the State. These should be applied in any type of judicial process; however they have not been able to position itself as a type of main notification, since its character is still optional and subsidiary. We analyze their development in judicial processes, emphasizing their evolution within the administrative and verbal process. Likewise, to the subsequent collection, analysis and interpretation of doctrine, legislation, jurisprudence and comparative law corresponding to the subject, we will have a compendium of characteristics, certain principles, possible security mechanisms and special legislation. Although it concerns the security offered for its use, by the little diligence of the State and the necessity of a secure system to implement them to the different processes.
\end{abstract}

Keywords: Electronic notifications, implementation, fundamental rights, verbal process, administrative process.

\title{
Introducción
}

A continuación se abordará la implementación de las notificaciones electrónicas (NE de ahora en adelante) en los procesos judiciales como el verbal, entendiéndose incluido el verbal sumario, y el administrativo. A través de una investigación explicativa se estudiará su implementación a nivel doctrinario, legislativo y jurisprudencial, haciendo una breve reseña del derecho comparado, así también los efectos de la implementación de las NE a nivel procesal, enfatizando las características específicas que poseen.

El artículo circunda en torno a la pregunta ¿Cómo se ha llevado a cabo la implementación de las $\mathrm{NE}$ en el proceso verbal y administrativo a partir de la doctrina, legislación, jurisprudencia y derecho comparado? Dado que la notificación personal, por medio electrónico, es subsidiaria pues solo es válida si el interesado acepta ser notificado de esa manera ${ }^{1}$. Pero a pesar de su uso facultativo se debe garantizar la integridad, inalterabilidad y confidencialidad

CARRILLO PARADA, José Rafael. 2013. "Procedimiento para las notificaciones administrativas”. Revista Electrónica de la facultad de Derecho. Vol.1, Núm. 1. (2013), 
del mensaje de datos ${ }^{2}$, por medio del cual se realiza la NE, para poder aplicar el principio de equivalente funcional. Lo anterior aunado a los avances legislativos, paulatinos y apropiados, que se deben realizar, en aras de lograr el carácter principal de las NE por diferentes medios como el correo o dirección electrónica, bases de datos, entre otros, y mancomunadamente a las nuevas tecnologías, que impulsarán la implementación y uso de las NE en todos los procesos judiciales, ya que por medio del uso de skype se podría realizar una notificación en estrados de manera virtual.

Igualmente, se abordará la implementación de las NE, teniendo en cuenta sus principios básicos y evolución en los procesos judiciales, como el verbal y administrativo. Se hará lo correspondiente con los beneficios, y obligaciones, que contrae su implementar, si bien las condiciones de seguridad ofrecidas actualmente no resultan idóneas. Por ello abrimos la puerta a un sistema de mensajería con operabilidad y seguridad adecuada, que sería oportuno para ampliar el uso de las NE.

\section{Necesidad de implementar las NE junto a las nuevas tecnologías}

Es importante aclarar que la implementación de las NE, como consecuencia de la implementación de las nuevas tecnologías, no representa un simple capricho o esfuerzo banal por parte del poder público en nuestra administración de justicia.

Actualmente la administración de justicia en Colombia, se encuentra colapsada, obtiene muy bajas calificaciones en materia de tiempos de decisión de controversias ${ }^{3}$, como en materia de percepción por parte de los usuarios del sistema ${ }^{4}$. Por lo cual es menester un cambio en el desarrollo del proceso que

Universidad de Santander, UDES Cúcuta. Véase: http://service.udes.edu.co/revistas/ index.php/Lex-UDES/article/P5.pdf. [Consultado el 01 de abril de 2017].

2 DÍAZ GARCÍA, Alexander. Las notificaciones electrónicas judiciales en Colombia: El notario electrónico (firmado electrónicamente). Enlace [online]. 2008. Vol.5. N² 2, pp. 131-138. Véase: [http://www.scielo.org.ve/scielo.php?script=sci_arttext\&pid=S16907515 2008000200008\&lng=es\&nrm=is]. ISSN 1690-7515. [Consultado el 02 de Abril de 2017].

3 Doing Business. “Cumplimiento de Contratos". Banco Mundial. Junio de 2016. Véase: [http://espanol.doingbusiness.org/data/exploretopics/enforcing-contracts], consultado el 21 de Noviembre de 2016.

4 World Justice Project. "Rule of Law Index”. 2014. Véase: http: //worldjusticeproject.org/ sites/default/files/files/wjp_rule_of_law_index_2014_report.pdf, [consultado el 25 de Noviembre de 2016]. 
permita mejoras en la decisión de controversias y percepción que tienen las personas acerca de aquella.

La implementación de las NE permitirá mejorar los aspectos anteriores pues ofrece mecanismos eficientes para superar demoras en el trámite de los procesos, levantando diversas barreras como las geográficas, lingüísticas, físicas, económicas, entre otras, de acceso a la administración de justicia proporcionando un mayor acceso a la misma, permitiendo proteger la tutela jurisdiccional efectiva ${ }^{5} \mathrm{y}$ haciendo más ágil el proceso.

Las TIC, entre ellas las NE, pueden aportar a la reducción de los tiempos en los procesos judiciales, para mejorar la administración del despacho judicial, reducir el uso de papel y del espacio disponible en los edificios destinados a oficinas judiciales, lo cual debe ir aunado a un cambio de lógica del juicio por uno basado en la oralidad e inmediación ${ }^{6}$, como se ha tratado de hacer en nuestra administración de justicia, con diferentes leyes como las 1564 de 2012 y 1437 de 2011.

El uso de las nuevas tecnologías en la administración de justicia puede suponer importantes beneficios a su funcionamiento pues permite; ahorrar tiempo, trabajo, obtener mayor información y transparencia sobre el funcionamiento de la justicia, y ofrecerla de manera eficaz; además las partes pueden relacionarse directamente con la justicia, lo que les puede facilitar el acceso a la misma y obtener mayor eficiencia en el proceso, ahorro de tiempo, disminución de costos y una justicia de mejor calidad. ${ }^{7}$ En igual sentido, al implementar las nuevas tecnologías se daría aplicación al uso de audiencias virtuales, las cuales implicarían la posibilidad de realizar una notificación en estrados de manera virtual, y así aumentar el acceso a la administración de justicia eliminando barreras geográficas.

De igual forma, teniendo en cuenta la Carta Iberoamericana de Gobierno Electrónico, usar las tecnologías de la información y la comunicación en los órganos de la administración pública fomenta la mejora sustancial de la infor-

5 Pájaro Moreno Nicolás. "Las Tics al servicio del proceso". Memorias del XXXV Congreso Colombiano de Derecho Procesal. Cartagena. Septiembre de 2014. Véase: [https:// www.academia.edu/8867673/Las_TIC_al_servicio_del_proceso], consultado el 17 de Noviembre de 2016.

6 LILLO LOBOS, Ricardo. "El uso de las nuevas tecnologías en el sistema judicial: experiencias y precauciones”. II Justicia. 2011. Véase: http://www.iijusticia.org/docs/LOBOS. pdf. [Consultado Abril 2 de 2017].

7 CERRILLO, Agustí. "E-justicia: las tecnologías de la información y el conocimiento al servicio de la justicia iberoamericana en el siglo XXI”. IDP Revista de Internet, Derecho y Política. Número 4. 2007. [Consultado el 3 de Abril de 2017]. 
mación, oportunidad, eficiencia y eficacia de la gestión pública, generando un mejor servicio. ${ }^{8}$

Lo anterior se tornaría más factible con la creación de un sistema de mensajería con operabilidad y seguridad adecuada el cual, como su nombre lo indica, debe ofrecer unas condiciones mínimas de seguridad para la apropiada transferencia de los mensajes de datos y brindar mayor certeza al ciudadano al usar las NE en el proceso.

\section{Notificaciones electrónicas a nivel doctrinario}

Los mensajes de datos deben recibir el mismo tratamiento de los documentos físicos cuando aquel sea completo, auténtico, confiable, sincronizado y seguro. De igual forma el artículo 5 de la Ley 527 de 1999 prohíbe negar efectos jurídicos, validez o fuerza obligatoria a información, por el solo hecho de que sean mensajes de datos?.

Para una debida implementación de las TIC se debe desarrollar en dos planos estrechamente interconectados: el de los usuarios externos ${ }^{10}$ de la administración de justicia, de los interesados en el proceso, y el de los usuarios internos ${ }^{11}$, los funcionarios y empleados que deben trabajar en aras de las funciones judiciales ${ }^{12}$.

8 MACARENO LÓPEZ, Roger Adolfo (2012) "notificación electrónica de los actos administrativos: ¿hay violación del debido proceso?”. Revista Iter Ad Veritatem 10. Universidad Santo Tomás, Tunja. Véase: http://revistas.ustatunja.edu.co/index.php/ iaveritatem/article/view/559/379 [Consultado el 31 de marzo de 2017].

9 UMAÑA CHAUX, Andrés. "Algunos comentarios sobre el principio del equivalente funcional en la ley 527 de 1999". Revista de derecho, comunicaciones y nuevas tecnologías N 1․2005. Bogotá D.C., p. 78. Disponible en: https://derechoytics.uniandes.edu.co/compo nents/com_revista/archivos/derechoytics/ytics93.pdf [Consultado en 8, febrero, 2016]

10 Los usuarios externos son todas las personas que acuden a la administración de justicia para obtener de ella la definición o la ejecución coactiva de sus derechos. es necesario que el diseño de las herramientas tecnológicas se base en la experiencia y necesidades de los jueces, de los abogados litigantes y de las mismas partes, para explotar al máximo sus posibilidades, y generar un valor agregado en su uso, y para que sirva de un soporte efectivo a otros aspectos esenciales de los nuevos esquemas procesales de oralidad.

11 Los usuarios internos son quienes administran justicia, y el personal adicional que soporta, desde escenarios estratégicos o de apoyo, el cumplimiento de la función jurisdiccional. En términos generales, se trata de los funcionarios y empleados de la Rama Judicial. Las funciones administrativas a través de la correcta implementación de las TIC, pueden ser sistematizadas y automatizadas, en aras de facilitar el trabajo de los empleados de la administración de justicia.

12 PÁJARO MORENO, Nicolás. "Las TIC al servicio del proceso". Memorias de XXXV Congreso Colombiano de Derecho Procesal. ICDP/UniLibre. Septiembre de 2014. 
Para implementar las NE se requiere el uso de un sistema de seguridad, o entidades de certificación, que son entidades facultadas, conforme a la ley, para emitir certificados en relación a la recepción, integridad e inalterabilidad de los mensajes de datos y otras funciones que garantizarán su integridad, inalterabilidad y confidencialidad de las NE, lo cual es menester para otorgarle carácter de personal ${ }^{13}$.

\subsection{Principios fundamentales para la implementación de las notificaciones electrónicas}

En la implementación de las NE se deben tener en cuenta los principios de neutralidad económica y equivalente funcional ${ }^{14}$, los cuales permiten un igual tratamiento entre los documentos físicos y mensajes de datos, independiente del soporte que estos usen. A continuación se los explicará brevemente:

Principio de equivalente funcional: Permite dar igual tratamiento a documentos electrónicos y físicos, con la condición de cumplir las características de integridad, rastreabilidad, seguridad, autenticidad, entre otras ${ }^{15}$.

Principio de neutralidad tecnológica: Permite la aplicación analógica del derecho cuando la actividad realizada es exactamente la misma independientemente del soporte utilizado ${ }^{16}$, busca que la actividad no este ligada a ninguna tecnología.

Para implementar las NE se debe garantizar la inalterabilidad, confiabilidad e integridad de los mensajes de datos, acorde a los anteriores principios, junto a

Disponible en: https://www.academia.edu/8867673/Las_TIC_al_servicio_del_proceso [Consultado 2 Enero de 2017]

13 DÍAZ GARCÍA, Alexander. "Colombia. Aspectos fundamentales del proceso, desde la óptica de las nuevas tecnologías. El documento electrónico judicial en Colombia”. Revista de derecho informático $\mathrm{N}^{\circ}$ 116. 2008 Lima, p. 2. [Consultado Noviembre 18 de 2016].

14 FLÓREZ, German Darío. "La validez jurídica de los documentos electrónicos en Colombia a partir de su evolución legislativa y jurisprudencia”. Revista Verba Iuris N 31.2014 Bogotá D.C., pp. 46-51. Disponible en: http://www.unilibre.edu.co/verbaiuris/31/ la-validez-juridica-de-los-documentos-electronicos-en-colombia-a-partir-de-su-evolucion-legislativa-y-jurisprudencial.pdf [Consultado en 28 de marzo de 2016].

15 Ibídem.

16 ALONSO ESPINOSA, Carles. "La información en la red y el principio de neutralidad tecnológica: la libertad de expresión y la difusión de información administrativa". Revista derecho del Estado $\mathrm{N}^{\circ}$ 22. Junio de 2009. Bogotá D.C., p. 87. Disponible en: http://revistas. uexternado.edu.co/index.php/derest/article/view/480/459 [Consultado Agosto, 9, 2016] 
herramientas y medios para salvaguardar los mismos. Así las NE ampliaran su margen de uso y confiabilidad dentro de la administración de justicia y por parte de los particulares, por lo cual los esfuerzos deben dirigirse a salvaguardar los mismos.

\section{Notificaciones electrónicas a nivel legislativo}

Notificación es el acto mediante el cual se pone en conocimiento de los sujetos procesales el contenido de las providencias proferidas en un proceso, tiene como finalidad garantizar los derechos de defensa y de contradicción como nociones integrantes del debido proceso por lo cual se deben cumplir diferentes requisitos y garantías para lograr su debida realización. ${ }^{17}$

Las NE, representan una de las maneras para realizar la notificación ${ }^{18}$ $y$, abren la posibilidad de que las partes en un proceso judicial puedan ser informadas de la totalidad, o parte, de los actos procesales con el uso de mecanismos técnicos o informáticos como el correo electrónico. ${ }^{19}$ Aquellas se realizan a través de mensajes de datos, los cuales son, la información generada, enviada, recibida, archivada o comunicada por medios electrónicos, ópticos o similares, como pudieran ser, entre otros, el intercambio electrónico de datos (EDI), el correo electrónico, el telegrama, entre otros ${ }^{20}$, al mencionar "medios similares" refiere que los mensajes de datos no están exclusivamente destinados a las prácticas modernas de comunicación, sino que pretenden ser útiles para involucrar todos los adelantos tecnológicos futuros. ${ }^{21}$

Las NE permitirían agilizar los procesos y hacerlos más eficaces para superar la congestión imperante en aquellos; además permiten aplicar los

17 Colombia. Corte Constitucional. Sala plena. Sentencia de constitucionalidad $N^{\circ} 648$ del 20 de Junio de 2001. M.P. Marco Gerardo Monroy Cabra. Expediente D-3365.

18 Colombia. Congreso de la República. Ley 1564 del 12 de Julio de 2012. Artículo 291 numeral $3^{\circ}$; artículo 292.

19 QUINTERO NAVAS, Gustavo. "Contencioso administrativo y medios electrónicos: un gran paso hacia la modernización del ejercicio de la justicia administrativa". Revista de derecho, comunicaciones y nuevas tecnologías №6. Bogotá. 6 de diciembre de 2011. Universidad de los Andes, pp. 5-7. Véase: https://derechoytics.uniandes.edu. co/components/com_revista/archivos/derechoytics/ytics103.pdf [Consultado en 13, septiembre, 2016].

20 Colombia. Congreso de la República. Ley 527 del 18 de agosto 1999. Artículo $2^{\circ}$ literal A.

21 Colombia. Corte Constitucional. Sala plena. Sentencia de constitucionalidad $N^{\circ} 831$ del 8 de agosto de 2001. M.P. Tafur Galvis Álvaro. Expediente D-3371. 
principios de celeridad y economía procesal, por lo que desde $1996^{22}$ el Consejo Superior de la Judicatura debe propender su implementación, uso y desarrollo, con las nuevas tecnologías.

El Consejo Superior de la Judicatura siempre debe procurar el respeto y salvaguarda del derecho al debido proceso y publicidad, consagrado el primero por la Corte Constitucional como el conjunto de garantías previstas en el ordenamiento jurídico, a través de las cuales se busca la protección del individuo incurso en una actuación judicial o administrativa, para que durante su trámite se respeten sus derechos y se logre la aplicación correcta de la justicia ${ }^{23}$. El principio de publicidad consiste en dar a conocer, a través de publicaciones, comunicaciones o notificaciones, las actuaciones judiciales y administrativas a toda la comunidad, como garantía de transparencia y participación ciudadana, así como a las partes y terceros interesados en un determinado proceso para garantizar sus derechos de contradicción y defensa. ${ }^{24}$ Por lo cual se debe tener como premisa, en la implementación de las NE, la integridad, inalterabilidad y confidencialidad de los mensajes de datos, por los que se transfieren.

Las NE deben implementarse de manera segura, eficaz y eficiente; de manera conjunta con bases de datos, condiciones técnicas y capacitaciones adecuadas para igual fin. De gran ayuda, en aquel sentido, serían las entidades de certificación, las cuales son una persona jurídica, con autorización legal, facultadas para emitir certificados en relación con las firmas electrónicas de las personas, ofrecer o facilitar los servicios de registro y estampado cronológico de la transmisión y recepción de mensajes de datos, así como cumplir otras funciones relativas a la seguridad de comunicaciones basadas en las firmas electrónicas ${ }^{25}$ y se pueden usar en las NE. Cuentan con legislación, actividades ${ }^{26}$ y requisitos ${ }^{27}$ específicos, como consecuencia de la función especial de certificación que cumplen, y

22 Colombia. Congreso de la República. Ley 270 del 7 de marzo de 1996. Ley estatutaria de la administración de justicia.

23 Colombia. Corte Constitucional. Sala plena. Sentencia de constitucionalidad $N^{\circ} 980$ de 2010. $1^{\circ}$ de diciembre de 2010. M.P. Mendoza Martelo, Gabriel. Expediente D-8104.

24 Colombia. Corte Constitucional. Sala plena. Sentencia de constitucionalidad $N^{\circ} 12$ de 2013. 23 de Enero de 2013. M.P. González Cuervo, Mauricio. Expediente D-9195.

25 Colombia. Sala Administrativa del Consejo Superior de la Judicatura. Acuerdo $\mathrm{N}^{\circ}$ PSAA06-3334 del 2 de marzo de 2006. Reglamenta los medios electrónicos e informáticos en el cumplimiento de las funciones de la administración pública.

26 Colombia. Congreso de la República. Ley 527 del 18 de agosto 1999. Artículo 30.

27 Colombia. Presidente de la República de Colombia. Decreto 333 del 19 de febrero de 2014. Reglamenta el artículo 160 del Decreto-Ley 19 de 2012, define el régimen de aplicación de las entidades de certificación. 
proporcionan mayor seguridad al emitir y recibir mensajes de datos, medio por el cual se realizan las NE. Lo anterior para procurar una adecuada notificación, dado que esta trae consigo la efectividad de los principios de celeridad y eficacia de la función pública ${ }^{28}$.

\subsection{Notificaciones electrónicas en el Proceso Verbal}

En el proceso verbal, entiéndase incluido el verbal sumario, se espera un cambio en el uso de las NE respecto al proceso ordinario, dado que su uso fue precario en vigencia del Código de Procedimiento Civil, para lo cual cumple una función importante la entrada en vigencia del Código General del Proceso (C.G.P. de ahora en adelante).

A diferencia del Código de Procedimiento Civil, el C.G.P marcó la pauta para que todos los actos de notificación y comunicación del proceso se puedan llevar a través de mensajes de datos, sin importar la tecnología en boga de cada momento, ni las plataformas que decida implementar quien administra la jurisdicción ${ }^{29}$, otorgándole un enfoque progresivo al proceso verbal, como consecuencia de prepararlo a las diferentes condiciones técnicas, las cuales puede preverse que cambiarán.

En efecto, el C.G.P. no se limitó a vaticinar la posibilidad de que diversas formas de notificación se realicen a través del correo electrónico o de la publicación en bases de datos abiertas al público; en su artículo 103 estipulo que puede hacerse uso de cualquier otro mecanismo de envío, transmisión, acceso y almacenamiento de mensajes de datos siempre que garanticen la autenticidad e integridad de intercambio o acceso de información ${ }^{30}$, como podrían ser aplicaciones diseñadas para dispositivos móviles, sistemas de mensajería instantánea ${ }^{31}$, entre otros. Pues los medios electrónicos permiten el cumplimiento de los fines del Estado en concordancia a los principios establecidos por el C.G.P., como la eficacia, celeridad, economía, entre otros. ${ }^{32}$

28 Colombia. Corte Constitucional. Sentencia de Tutela N ${ }^{\circ} 210$ de 2010. 23 de Marzo de 2010. M.P. Henao Pérez, Juan Carlos. Expediente T. 2.367.072.

Ibíd.

30 Colombia. Congreso de la República. Ley 1564 del 12 de julio de 2012. Artículo 103 parágrafo $3^{\circ}$.

31 PÁJARO MORENO, Nicolás. "Las TIC al servicio del proceso". Memorias de XXXV Congreso Colombiano de Derecho Procesal. ICDP/UniLibre. Septiembre de 2014. Disponible en: https://www.academia.edu/8867673/Las_TIC_al_servicio_del_proceso [Consultado 2 Enero de 2017].

32 PEÑA VALENZUELA, Daniel. De la firma manuscrita a las firmas electrónica y digital. Edición 2015. Bogotá D.C. Universidad Externado de Colombia., p. 199. 
De igual manera equipara el valor demostrativo de los documentos físicos con los mensajes de datos, ${ }^{33}$ como las NE, por lo cual no se puede negar eficacia, validez o fuerza obligatoria y probatoria a información en forma de un mensaje de datos, solo por ser un mensaje de datos. ${ }^{34}$

El C.G.P. otorga a las NE un carácter facultativo, de manera implícita pero acentuada. Solo permite hacer que estas, en este caso se entenderá como personal, cuando se conozca la dirección electrónica de quien deba ser notificado ${ }^{35}$, pues las personas naturales son quienes deben suministrar al juez su dirección de correo electrónico ${ }^{36}$ de manera voluntaria, ya que en caso contrario no se podrá realizar la NE.

El C.G.P. ofrece diferentes garantías para la implementación de las NE y las nuevas tecnologías mediante obligaciones para la administración de justicia y las partes; el art. 78, numeral 14, reza que es obligación de las partes remitir copia de los memoriales presentados en el proceso, excepto las medidas cautelares, al correo electrónico de la contraparte cuando hubiese suministrado uno; el art. 82, parágrafo segundo, permite que las demandas presentadas a través de mensajes de datos no necesiten firma digital para su validez; el art. 122 permite que los memoriales sean enviados de manera electrónica; el art. 244 presume auténticos los mensajes de datos, respetando el principio de equivalencia funcional de los mensajes de datos; el art. 291 numeral segundo, impone la obligación a las personas jurídicas de derecho privado y los comerciantes inscritos en el registro mercantil de registrar en la Cámara de Comercio o en la oficina de registro correspondiente del lugar donde funcione su sede principal, sucursal o agencia, la dirección física y otra electrónica donde recibirán notificaciones judiciales; el art. 291 reza que cuando se conozca la dirección electrónica de quien deba ser notificado, la comunicación podrá remitirse por el Secretario o el interesado por medio de correo electrónico. Se presumirá que el destinatario recibió la comunicación cuando el iniciador recepcione acuse de recibo. ${ }^{37}$

El Consejo Superior de la judicatura intentó avanzar en el tema, al expedir el Acuerdo No. PSAA06-3334, y estableció pautas sobre el uso de medios electrónicos e informáticos en el cumplimiento de las funciones de la administración de justicia; sin embargo, se limitó a desarrollar definiciones y asignar la

33 Ibídem.

34 Colombia. Congreso de la República. Ley 527 de agosto 8 de 1999. Artículo 10.

35 Colombia. Congreso de la República. Ley 1564 del 12 de Julio de 2012. Artículo 291, numeral $3^{\circ}$.

36 Ibídem. Numeral $2^{\circ}$.

37 Colombia. Congreso de la República. Ley 1564 del 12 de Julio de 2012. Artículo 291, numeral $3^{\circ}$. 
posibilidad de realizar algunos actos de comunicación por mensajes de datos, pero sin carácter vinculante.

Por último se debe señalar un avance del Código de Procedimiento Civil, cuando define como documento todo objeto mueble que tenga carácter representativo o declarativo ${ }^{38}$, definición que integra las grabaciones de audio, vídeo y los mensajes de datos ${ }^{39}$, lo cual abrió la puerta para el uso de los últimos en el proceso civil.

\subsection{NE en el proceso administrativo y la Administración Pública}

Las NE han presentado un desarrollo paulatino en la administración pública, desde $1995^{40}$, cuando las entidades públicas debían habilitar sistemas de transmisión electrónica de datos, como un sistema de correo, para que los usuarios pudieran enviar y recibir información en sus actuaciones ante la administración, esto sin poder restringir a los particulares el uso de tecnologías para su archivo documental.

En el proceso administrativo, las autoridades pueden notificar sus actos a través de medios electrónicos, si el administrado aceptó ese medio de notificación. Pero durante el desarrollo de la actuación el interesado podrá solicitar a la autoridad que las notificaciones sucesivas no se realicen por medios electrónicos, sino conforme con los otros medios previstos ${ }^{41}$, si el administrado acepta la NE esta se tomará carácter de personal ${ }^{42}$-reiteración del carácter facultativo- pero cuando se haga NE debe adjuntarse el texto completo del actuación, o en caso contrario será invalida. ${ }^{43}$

El acto administrativo solo tiene plenitud cuando se lo hace conocer al administrado, ahí se perfecciona, no produce efectos jurídicos antes. Por lo que para la efectividad del acto administrativo debe realizarse una adecuada NE, que

38 Colombia. Congreso de la República. Ley 1400 del 21 de septiembre de 1970. Artículo 251.

39 Colombia. Corte Suprema de Justicia. Sala de casación civil. Sentencia de 16 de diciembre de 2010. M.P. Pedro Octavo Munar Cadena. Expediente 11001311000520040107401. Colombia. Congreso de la República. Decreto 2150 del 05 de Diciembre de 1995. Art. 26

41 Colombia. Congreso de la República. Ley 1437 del 18 de enero de 2011. Artículo 56.

$42 \quad$ Ibídem. Artículo 67.

43 Colombia. Consejo de Estado. Sala de lo Contencioso Administrativo. Sección segunda. C.P. Vergara Quintero, Luis Rafael. Marzo 11 de 2013. Radicación: 25000-23-37-0002012-00459-01(AC). 
también debe garantizar el debido proceso, y los principios de celeridad, eficacia y publicación. ${ }^{44}$

Un avance importante en la implementación de las NE es la obligación de las entidades públicas, y particulares que cumplan función pública, de tener un buzón de correo electrónico exclusivamente para recibir NE, las cuales se entenderán como personales ${ }^{45}$, lo anterior se ejemplifica con el correo de la Agencia Nacional de Defensa Jurídica del Estado ${ }^{46}$, la secretaría general de la Alcaldía de Bogotá ${ }^{4}$.

En materia de Aduanas y de Control de Cambios, los actos administrativos que decidan recursos y las actuaciones que deban notificarse se realizarán a la dirección electrónica o sitio electrónico que asigne la DIAN a los contribuyentes que opten por la $\mathrm{NE}^{48}$. En materia tributaria las $\mathrm{NE}$ se entenderá surtida en el momento que se produzca el acuse de recibido en la dirección o sitio electrónico asignado ${ }^{49}$. En relación a lo anterior, la Corte Constitucional ha declarado que consagrar formas electrónicas de notificación por aviso electrónico como mecanismo subsidiario para suplir trámites de notificación infructuosos, no resulta violatorio del debido proceso ni del derecho de defensa ${ }^{50}$, la impugnación puede ser enviada al correo electrónico del ente de control, que debe recepcionarlo y tramitarlo de manera adecuada y similar a un escrito. ${ }^{51}$ En el proceso contencioso administrativo se presumirá que el destinatario ha recibido la notificación cuando el iniciador recepcione acuse

44 MACARENO LÓPEZ, Roger Adolfo (2012) "notificación electrónica de los actos administrativos: ¿hay violación del debido proceso?”. Revista Iter Ad Veritatem 10. Universidad Santo Tomás, Tunja. Véase http://revistas.ustatunja.edu.co/index.php/ iaveritatem/article/view/559/379. [Consultado Abril 2, 2017].

45 Colombia, Congreso de la República. Ley 1437 del 18 de enero de 2011. Artículo 197.

46 Colombia. Agencia Nacional de Defensa Jurídica del Estado. Circular Externa 03 del 3 de junio de 2016. Notificación judicial por medios electrónicos.

47 Colombia. Secretaria general de la alcaldía de Bogotá. Circular Externa 86 del 14 de agosto de 2012.

48 Colombia. Congreso de la República. Ley 1111 del 27 de diciembre de 2006. Modifica el estatuto tributario de los impuestos administrados por la Dirección de Impuestos y Aduanas Nacionales.

49 Colombia. Dirección de Impuestos y Aduanas Nacionales. Concepto 5473 del 12 de marzo de 2016.

50 Colombia. Corte Constitucional. Sala plena. Sentencia de constitucionalidad $N^{\circ} 016$ del 23 de enero de 2013. M.P. Mendoza Martelo, Gabriel. Expediente N D-9091.

51 Colombia. Corte Constitucional. Sala sexta de revisión. Sentencia de tutela $\mathrm{N}^{\circ} 286$ del 20 de mayo de 2013. M.P. Pinilla Pinilla, Nilson. Expediente N T- 3748679. 
de recibo o se pueda por otro medio constatar el acceso del destinatario al mensaje ${ }^{52}$.

De igual manera los vigilados de la superintendencia de salud pueden usar las NE en los actos administrativos, para lo cual sus representantes legales deben manifestar expresamente su voluntad de usarlas ${ }^{53}$-reiteración al carácter facultativo de las NE-.

Es claro pues que las NE han encontrado su mayor desarrollo a nivel nacional en la administración pública, pero infortunadamente la falta de regulación legal y la delegación excesiva al Consejo Superior de la Judicatura no han permitido avances trascendentales ${ }^{54}$.

\subsection{Aplicación y uso de las notificaciones electrónicas y las nuevas tecnologías}

La Rama Judicial tiene en funcionamiento el software Siglo XXI ${ }^{55}$ el cual permite; tener un número único de radicación, facilitar el control sobre los términos, el reparto, ubicación de los expedientes con información directa de los despachos y conocer el estado del proceso a través de un computador, aunque el mismo sólo funciona en la medida en que esté instalado en el computador del despacho respectivo, y que además funciona a través de redes locales, lo cual genera que cada despacho cuente con una versión distinta del programa, a veces con problemas de interoperabilidad, que la disponibilidad de la información para el ciudadano dependa de la estabilidad de la red local, y que las consultas dependan de la ubicación física de los despachos judiciales ${ }^{56}$, lo cual lo convierte

52 Colombia. Congreso de la República. Ley 1437 del 18 de enero de 2011. Código de Procedimiento Administrativo y de lo Contencioso Administrativo. Artículo 205.

53 Colombia. Superintendencia Nacional de Salud. Carta Circular 2 del 17 de Octubre de 2012.

54 PÁJARO MORENO, Nicolás. "Las TIC al servicio del proceso". Memorias de XXXV Congreso Colombiano de Derecho Procesal. ICDP/UniLibre. Septiembre de 2014. Disponible en: https://www.academia.edu/8867673/Las_TIC_al_servicio_del_proceso [Consultado 2 Enero de 2017].

55 Colombia. Sala Administrativa del Consejo Superior de la Judicatura. Acuerdo 1591 del 24 de Octubre de 2002. Se establece el sistema de información de gestión de procesos y manejo documental (Justicia Siglo XXI).

56 PÁJARO MORENO, Nicolás. "Las TIC al servicio del proceso". Memorias de XXXV Congreso Colombiano de Derecho Procesal. ICDP/UniLibre. Septiembre de 2014. Disponible en: https://www.academia.edu/8867673/Las_TIC_al_servicio_del_proceso [Consultado 2 Enero de 2017]. 
en ineficiente. En el caso de las NE, el software Siglo XXI resulta inocuo, por lo cual, se torna indispensable un sistema de operabilidad y seguridad adecuada, y un modo de hacerlo sería una red de mensajería con cifrado de extremo a extremo, que permita la interacción interna de la administración de justicia y los particulares de manera segura.

La Rama Judicial también cuenta con el sistema de gestión de correspondencia SIGOBius ${ }^{57}$, el cual tiene como objetivo realizar de forma integral todas las operaciones inherentes a la adecuada gestión de la correspondencia interna, aquella que se inicia en la institución o como respuesta a una correspondencia externa; su elaboración y posterior despacho a destinatarios internos o externos $^{58}$.

Deigual manera todos los juzgados de la Rama Judicial cuentan, por obligación, con un correo electrónico con dominio denominado @notificaciones.ramajudicial.gov.co, para realizar NE, cuando el interesado expresamente manifieste querer ser notificado de esta manera, lo cual reitera la calidad facultativa con la que cuentan las NE.

En el proceso una NE puede tener valor probatorio, para valorar su fuerza probatoria se deben tener en cuenta las reglas de la sana crítica y demás criterios reconocidos legalmente para la apreciación de las pruebas, ${ }^{59}$ por lo cual trasciende la confiabilidad de la forma en que se haya; generado, archivado o comunicado el mensaje, conservado la integridad de la información, en que se identifique a su iniciador y cualquier otro factor pertinente, por lo cual es pertinente la creación de un sistema de operabilidad y seguridad adecuada para la transferencia de mensajes de datos, pues si no se realiza se puede afectar la fuerza probatoria de las NE.

Es primordial resaltar que una NE se entiende debidamente surtida desde el momento en que el destinatario recibe efectivamente el mensaje de datos contentivo del acto $^{60}$, lo cual significa que sólo en ese momento y no antes,

57 Colombia. Sala Administrativa del Consejo Superior de la Judicatura. Acuerdo $\mathrm{n}^{\circ}$ PSAA11-8707 de octubre 3 de 2011 y Acuerdo nº. PSAA14-10163 de junio 16 de 2014.

58 Colombia. Sala Administrativa del Consejo Superior de la Judicatura. "procedimiento para la gestión adecuada de la correspondencia interna a través de SIGOBius". Disponible en: http://sistemagestioncalidad.ramajudicial.gov.co/modelocsj/archivos/P-AGD-05/ PROCEDIMIENTO\%20PARA\%20LA\%20GESTION\%20ADECUADA\%20DE\%20 LA\%20CORRESPONDENCIA\%20INTERNA\%20A\%20TRAV\%C3\%89S\%20DE\%20 SIGOBius.pdf [Consultado 30 de enero de 2017].

59 Colombia. Congreso de la República. Ley 527 del 18 de agosto de 1999. Artículo 11.

60 Colombia. Congreso de la República. Ley 1437 del 18 de enero de 2011. Artículo 56. 
aquella resulta jurídicamente oponible, siendo el recibo de la comunicación el referente válido para contabilizar los términos en el proceso ${ }^{61} \mathrm{o}$ de recursos, pues en Colombia existe la posibilidad, a nivel administrativo, de usar las NE respecto a resoluciones de apertura de investigación, las que ponen fin a la actuación y las que deciden los recursos en la vía gubernativa ${ }^{62}$. Consecuentemente en el proceso verbal todas las actuaciones judiciales se pueden realizar a través de mensajes de datos ${ }^{63}$, lo cual es de carácter facultativo y solo se entienden aceptas de manera expresa ${ }^{64}$ o cuando se proporciona el correo electrónico.

Un modelo a seguir es el sistema MARCO@Online el cual es el portafolio de servicios electrónicos que optimiza la comunicación y la interacción entre las partes y el centro de arbitraje y conciliación MARCO, a través de internet. Permite radicar un caso, efectuar los pagos correspondientes, radicar y consultar documentos, entre otras posibilidades. Habilita la comunicación de dos vías entre los profesionales de MARCO y clientes y abogados. El sistema comenzó a ofrecerse en marzo de 2010 y el mejoramiento e incorporación de funcionalidades se ha venido realizando de manera gradual y actualmente los resultados dan cuenta de ahorros significativos de costos y tiempos de desplazamiento inherentes a una o varias reuniones presenciales entre las partes y los conciliadores o árbitros, transparencia, agilidad en los procesos y en general optimización y eficiencia en los servicios prestados a sus clientes. ${ }^{65}$

Las nuevas tecnologías asisten al derecho con videoconferencias ${ }^{66}$, el aula virtual de la escuela judicial "Rodrigo Lara Bonilla" ${ }^{67}$, Bogotá jurídica digital ${ }^{68}$, entre otras. En concordancia, realizar audiencias por medio de videoconferencias

${ }^{61}$ Colombia. Corte Constitucional. Sala plena. Sentencia de constitucionalidad $\mathrm{N}^{\circ} 980$ del 1 de diciembre de 2010. M.P. Mendoza Martelo, Gabriel. Expediente N ${ }^{\circ}$ D-8104.

62 Colombia. Congreso de la República. Ley 1340 del 24 de julio de 2009. Artículo 23.

63 Colombia. Congreso de la República. Ley 1564 del 12 de julio de 2012. Artículo 103.

64 Colombia. Congreso de la República. Ley 1437 del 18 de enero de 2011. Código de Procedimiento Administrativo y de lo Contencioso Administrativo. Artículo 205.

65 Corporación excelencia en la justicia. E-Justicia, experiencias internacionales y colombianas en el uso de las TIC para el mejoramiento de la administración de justicia. Tercera versión. 2013. Véase: http://www.cej.org.co/files/Experiencias_TIC-2013.pdf. [Consultado Abril 1 de 2017].

66 Corporación excelencia en la justicia. E-Justicia, experiencias internacionales y colombianas en el uso de las TIC para el mejoramiento de la administración de justicia. Tercera versión. 2013. Véase: http://www.cej.org.co/files/Experiencias_TIC-2013.pdf. [Consultado Abril 1 de 2017].

67 Véase: <http://aula.ejrlb.net/inicio>.

68 Véase: <http://www.cej.org.co/index.php/159-seccionpremio/nominados-3er-premio/ 2423-bogota-juridica-digital> 
permitiría innovar con la materialización de una notificación por estrados de manera virtual.

\subsection{Excepción del carácter facultativo de las notificaciones electrónicas}

Las NE ostentan un carácter facultativo, pero hay excepciones como en el registro mercantil, las entidades públicas, o privadas que cumplan funciones públicas, y el Ministerio Público al actuar ante lo contencioso administrativo o las autoridades en general. En el registro mercantil el administrado debe proporcionar la dirección electrónica, personas jurídicas de derecho privado y los comerciantes inscritos en el registro mercantil ${ }^{69}$, y no se hace extensiva la posibilidad a los usuarios de no ser notificado de manera electrónica ${ }^{70}$. Las entidades públicas, privadas que cumplan funciones públicas y el Ministerio Público que actúe ante lo contencioso administrativo, deben tener un buzón de correo electrónico exclusivo para recibir NE. ${ }^{71}$ De igual forma, toda autoridad debe tener, mínimo, una dirección electrónica ${ }^{72}$. En concordancia a lo anterior, lo esfuerzos legislativos deben ir encaminados a transformar las NE en el medio principal de notificación, y que la subsidiariedad radique en otro tipo de notificaciones, pues así se lograran diversos beneficios en el proceso mencionados previamente, como su agilización, mayor acceso, eficacia, entre otros. Lo cual encuentra sustento dentro de los casos mencionados al inicio de este acápite, pues los mismos han sido exitosos y han logrado una eficiente aplicabilidad.

\section{Notificaciones electrónicas a nivel jurisprudencial}

Las NE han tenido un amplio desarrollo a través de la jurisprudencia de la Corte Suprema de Justicia, Corte Constitucional y Consejo de Estado, subsanando vacíos, ambigüedades y vaguedades normativas, señalando el proceder para su uso e implementación adecuada y las características que deben tener para ser válidas.

69 Colombia. Congreso de la República. Ley 794 del 08 de enero de 2003. Artículo 29․

70 FLÓREZ, Germán Darío. "La validez jurídica de los documentos electrónicos en Colombia a partir de su evolución legislativa y jurisprudencial". Revista Verba Iuris 31. 2014. Bogotá D.C., pp. 43, 71. Véase http://www.unilibre.edu.co/verbaiuris/31/ la-validez-juridica-de-los-documentos-electronicos-en-colombia-a-partir-de-su-evolucion-legislativa-y-jurisprudencial.pdf [Consultado 28 marzo de 2016].

${ }^{71}$ Colombia. Congreso de la República. Ley 1437 de enero 18 de 2011. Artículo 197º.

72 Ibídem. Artículo 60. 


\subsection{NE frente a la notificación personal y los documentos físicos}

La notificación tiene como finalidad garantizar el conocimiento de la existencia de un proceso o actuación administrativa y su desarrollo, respetando los principios de publicidad, contradicción y en especial, prevenir que alguien pueda ser condenado sin ser oído ${ }^{73}$.

El legislador ha establecido la notificación personal como principal, por las garantías que brinda, siendo la forma de notificación más segura con la cual cuenta la administración de justicia aunque también ha regulado formas de notificación de carácter subsidiario, donde se encuentra la NE que permite una notificación ágil, eficaz y económica ${ }^{74}$, la cual en determinados casos se puede tomar como personal. Aunque su carácter es subsidiario y facultativo, representa una posibilidad para mejorar el proceso como consecuencia de una comunicación elocuente y fluida entre las partes y la administración de justicia en el proceso.

La Corte Constitucional ha declarado que la NE, o por correo, representa un mecanismo adecuado, idóneo y eficaz, que garantiza el principio de publicidad y el debido proceso, dado que es una manera legítima de poner en conocimiento la actuación procesal o administrativa, a los interesados ${ }^{75}$-una muestra de ello es la opción de los vigilados de la Superintendencia de Puertos y Transporte para usarla.$-^{76}$

No se puede obviar que las NE se realizan por medio de mensajes de datos los cuales deben recibir el mismo tratamiento de los documentos consignados en papel, es decir, debe dársele igual eficacia jurídica acorde al principio de equivalencia funcional, por cuanto comportan los mismos criterios ${ }^{77}$. Aquel principio exige que se tenga en cuenta los requisitos de forma, fiabilidad, inalterabilidad y rastreabilidad, que son aplicables a los documentos físicos, con lo cual cumplen

73 Colombia. Corte Constitucional. Sala Quinta de Revisión. Sentencia de Tutela $\mathrm{N}^{\circ} 165$ del 12 de febrero de 2001. M.P. Hernández Galindo, José Gregorio. Expediente Nº T-353539.

74 Colombia. Corte Constitucional. Sala plena. M.P. Araujo Rentería, Jaime. Sentencia de Constitucionalidad $N^{\circ} 783$ del 18 de agosto de 2004.

75 Colombia. Corte Constitucional. Sala plena. Sentencia de constitucionalidad $\mathrm{N}^{\circ} 012$ del 23 de enero de 2013 M.P. González Cuervo, Mauricio. Expediente Nº D - 9195

76 Colombia. Superintendencia de Puertos y Transporte. Circular 16 del 28 de junio de 2012. Notificación Electrónica de Actos Administrativos Ley 1437 del 18 de enero de 2011.

77 Colombia. Corte Constitucional. Sala plena. Sentencia de constitucionalidad $N^{\circ} 662$ del 8 de junio de 2000. M.P. Morón Díaz, Fabio. Expediente N D-2693. 
los mensajes de datos y están en capacidad de brindar similares estándares que el papel, e incluso mayor confiabilidad y rapidez si cumplen los requisitos legales ${ }^{78}$.

\subsection{La ley 527 de 1999 desde una perspectiva jurisprudencial}

La ley 527 de 1999 regula el uso de los mensajes de datos y ha sido fundamental para que el contenido de las NE opere conforme al principio de equivalente funcional, otorgándoles carácter vinculante, en determinados casos, lo cual obliga a los funcionarios y empleados judiciales a darle igual tratamiento.

A través del tiempo ha tenido diversas interpretaciones, dejando en un limbo jurídico su aplicabilidad y uso esencialmente en los mensajes de datos como NE, hasta que la Corte Constitucional declaró que no se restringe a las operaciones comerciales sino que hace referencia en forma genérica al acceso y uso de los mensajes de datos, lo que obliga a una comprensión sistemática de sus disposiciones con el conjunto de normas que se refieren a este tema dentro de nuestro ordenamiento jurídico y en particular con las disposiciones que se han ocupado de esta materia ${ }^{79}$. Los juzgados, tribunales y corporaciones judiciales pueden utilizar cualesquiera medios técnicos, electrónicos, informáticos y telemáticos, para el cumplimiento de sus funciones y que los documentos emitidos por los citados medios, cualquiera que sea su soporte, tendrán la validez y eficacia de un documento original, sí garantizan la autenticidad, integridad y el cumplimiento de los requisitos exigidos por las leyes procesales. ${ }^{80}$

\subsection{Requisitos de seguridad de las notificaciones electrónicas}

Las NE representan una opción importante para dinamizar los diferentes procesos, pero como consecuencia de los diferentes riesgos en el uso de las tecnologías y los diferentes peligros informáticos se debe dotar a las NE de estándares y requisitos estrictos de seguridad para lograr la integridad, inalterabilidad, confiabilidad que se le exige a los mensajes de datos para darles el mismo tratamiento que a los documentos físicos ${ }^{81}$. Por lo anterior, reitero la necesidad

$78 \quad$ Ibíd.

79 Colombia. Corte Constitucional. Sala Plena. Sentencia de constitucionalidad $\mathrm{N}^{\circ} 831$ del 8 de agosto de 2001. M.P. Tafur Galvis, Álvaro. Expediente Nº D-3371.

80 Ibídem.

81 FLÓREZ, Germán Darío. "La validez jurídica de los documentos electrónicos en Colombia a partir de su evolución legislativa y jurisprudencia”. Revista Verba Iuris $N^{\circ} 31$. 2014 Bogotá D.C., pp. 46-51. Disponible en: http://www.unilibre.edu.co/verba iuris/31/ 
de un sistema de operabilidad y seguridad adecuada, del cual carece la administración de justicia, y el cual no se puede desatender por razones económicas, o de diversa índole, dado que se encuentra en juego derechos fundamentales, como el acceso a la justicia, debido proceso, etc.

Las NE en toda actuación administrativa o judicial, tendrán validez, eficacia y fuerza obligatoria, si el destinatario avala esta opción y suministra su dirección electrónica ${ }^{82}$, siendo vital la protección de los mismos para garantizar los derechos fundamentales de los usuarios. Por lo cual se indicaran algunos procedimientos, o medidas, que ayudarían a la seguridad de los mensajes de datos.

La Corte Suprema de Justicia ha enfatizado en el procedimiento de "sellamiento" del mensaje, aquel se condensa de forma algorítmica y acompaña al mensaje durante la transmisión, siendo recalculado al final de ella en función de las características del mensaje realmente recibido; de modo pues, que si el mensaje recibido no es exacto al remitido, el sello recalculado no coincidirá con el original y, por tanto, así se detectará que existió un problema en la transmisión y que el destinatario no dispone del mensaje completo ${ }^{83}$. En igual sentido, la tecnología actual permite que el emisor tenga la opción de conocer si el receptor abrió el buzón de correo electrónico y presumiblemente leyó el mensaje, para proteger la integridad del mensaje de datos. Ambas medidas, anteriormente mencionadas, son válidas para el sistema por el cual propugnamos en esta oportunidad para la adecuada transmisión de mensajes de datos. En igual sentido se podría hacer uso de entidades de certificación, como lo es certicámara a través de certimail, las cuales han tenido un adecuado funcionamiento y garantizarían mayor acceso y confiabilidad a los mensajes de datos. Lo anterior sin ir en contra de la presunción de autenticidad de los mensajes de datos, por el contrario, en aras de garantizar una adecuada notificación y una función jurisdiccional diáfana.

En este sentido, en el proceso civil mediante el software Justicia siglo XXI o gobierno en línea, al optar por las NE, se le exige al interesada hacer un pre-registro que lo habilitará, mediante el uso de contraseña o de certificado digital, para acusar el recibo de su notificación, conocer el texto de la decisión, los recursos que procedan y las condiciones para su ejercicio, teniendo la opción de recibir la misma información que tendría acudiendo al despacho, así pues

la-validez-juridica-de-los-documentos-electronicos-en-colombia-a-partir-de-su-evolucion-legislativa-y-jurisprudencial.pdf [Consultado en 28 de marzo de 2016].

82 Colombia. Corte Suprema de Justicia. Sala de Casación Penal. Sentencia del 9 de abril de 2009. M.P. Espinoza Pérez, Sigilfredo. Expediente $N^{\circ} 28909$ notificación electrónica de actos administrativos ley 1437 del 18 de enero de 2011.

83 Colombia. Corte Suprema de Justicia. Sala de casación Civil. M.P. Munar Cadena Pedro. Expediente $\mathrm{N}^{\circ} 11001311000520040107401$ del 16 de diciembre de 2006. 
está en igual condición jurídica respecto de sus posibilidades de defensa a través de recursos y acceso a la justicia ${ }^{84}$. A pesar de lo anterior, el software presenta deficiencias referentes a su interoperabilidad, información al usuario y eficacia, lo que obliga a mejorarlo o crear otra plataforma.

\section{Falencias, y posibilidades, en la implementación de las NE}

El presente acápite se presenta como una opinión personal. Se puede evidenciar que la implementación de las NE ha hecho hincapié en nuestra administración de justicia desde finales del siglo pasado, siendo de notar diferentes avances un poco tímidos en el tema por parte del legislador, la jurisprudencia y doctrina, pero de forma concreta no se han logrado implementar en la totalidad del territorio nacional.

Existen diversos factores que derivan en lo mencionado previamente como la poca diligencia, falta de capacitación, entre otros. Pero el factor de mayor relevancia es la falta de un sistema de seguridad adecuado para las NE, ya que no se brindan garantías con miras a su integridad, autenticidad y confidencialidad, a pesar de ser un requisito esencial para su adecuada implementación y funcionamiento, por lo que me permito proponer la creación de un sistema de mensajería con operabilidad y seguridad adecuada, el cual debe ser una plataforma que brinde un alto grado de seguridad en el envío y recepción de NE, el mismo podría usar sistemas de seguridad existentes en la actualidad como el sellamiento del mensaje, mensajes con cifrado de extremo a extremo, entre otros, para así garantizar un acceso a la justicia transparente, efectivo y eficaz, lo cual ampliaría el uso de las NE. Lo anterior se deriva de la trascendencia de una adecuada notificación en los procesos judiciales, pues se inmiscuyen derechos fundamentales, los cuales no se pueden pasar por alto, y mucho menos como consecuencia de una ineficaz labor estatal.

\section{Notificaciones electrónicas en el derecho comparado}

Diferentes países han realizado la implementación de las NE de manera eficaz y eficiente, por ello a continuación se realizará una breve reseña, de la implementación de las NE en España, el Tribunal Virtual de Nuevo León y Costa Rica.

84 Colombia. Consejo de Estado. Sala de consulta y Servicio Civil. Oficio N ${ }^{\circ} 234$. Radicado

N 1989. 19 de Marzo de 2010 C.P. Arboleda, Enrique José. 
Aunque solo trataré los casos anteriormente mencionados me permitiré mencionar que Holanda es el pionero en el uso de las TIC para el intercambio de información entre los tribunales, las partes y el público general. Y en Inglaterra, el sistema XHBIT permite distribuir automáticamente el registro electrónico de lo ocurrido en audiencia a las partes por teléfono móvil, e-mail, vía web, entre otros.

\subsection{España}

La administración pública debe de impulsar el empleo y la aplicación de las técnicas y medios electrónicos, informáticos y telemáticos para el desarrollo de su actividad, entre las que se encuentra los actos de notificación, desde el siglo pasado ${ }^{85}$. De igual forma, es una capacidad discrecional de las personas el uso de medios electrónicos en la comunicación con la administración pública, teniendo la capacidad de cambiar el medio de comunicación en cualquier momento, pero al igual que en Colombia existen excepciones donde es obligatoria la comunicación a través de medios electrónicos ${ }^{86}$.

Las partes en el proceso tienen derecho a la igualdad en el acceso electrónico a los servicios de la administración de justicia con independencia de sus circunstancias personales, a la garantía de seguridad y confidencialidad de los datos que figuren en los ficheros, sistemas y aplicaciones de la administración de justicia, entre otros, y se le confiere la obligación a las oficinas de información y atención al público de poner a disposición de las personas de forma libre y gratuita los medios e instrumentos precisos para ejercer los anteriores derechos. De igual manera, se crearon puntos de acceso electrónico disponibles a los ciudadanos para sus relaciones con la administración de justicia, teniendo la obligación legal de dotar de medios e instrumentos electrónicos necesarios para desarrollar la función eficientemente. ${ }^{87}$

85 España. Jefatura de Estado. Juan Carlos I Rey de España. Ley 30 del 26 de Noviembre de 1992. Artículo 45.1.

86 Una excepción son las personas jurídicas, las entidades sin personalidad jurídica, Notarios, mercantiles, apoderados de quien escogiere este tipo de comunicación y los empleados de la administración pública en uso de sus funciones. España. Jefatura de Estado. Ley 39 del 2 de octubre de 2015 "Procedimiento administrativo común de las administraciones públicas. Artículo 14".

87 España. Jefatura del Estado. Ley 18 del 11 de Julio de 2011. 


\subsection{Tribunal virtual de nuevo león en México ${ }^{88}$}

Se creó en el 2005 y funciona a través de su portal web, cuenta con el tribunal y seis juzgados virtuales a los cuales se tiene acceso desde cualquier teléfono inteligente. ${ }^{89}$ Es un sistema informático que permite administrar expedientes y ofrecer servicios de consulta a las partes y sus representantes legales a través de Internet. ${ }^{90}$

El tribunal se encuentra disponible en todo momento y desde cualquier parte del mundo, permitiendo revisar las actuaciones de los juzgados y el tribunal sin importar el lugar, a través de una computadora y un usuario que el tribunal se encarga de otorgar al iniciar el proceso. Además de contar con la ventaja de haberse desarrollado con una política de cero papel y permite que todos sus trámites se produzcan de manera virtual con el lleno de determinados requisitos. Demostrando que es posible implementar la nuevas tecnologías al proceso judicial.

El objetivo del Tribunal Virtual es apoyar la administración de justicia con seguridad y transparencia mediante una base de datos que se deberá mantener actualizada diariamente y su información se considera parte del archivo judicial, y en caso de presentar evidencia de alteración electrónica el administrador del sistema tomará las medidas pertinentes para impedir tales actos e informar al Tribunal Superior de Justicia y a los usuarios, quienes tendrán la posibilidad de retroalimentación para reportar cualquier circunstancia relacionanda con los servicios y funcionamiento del Tribunal Virtual, éste produce efectos de notificación, como medio informativo, a quien lo solicite y acepte usar este medio de manera expresa. ${ }^{91}$

\subsection{Costa Rica}

El sistema de Mensajería Judicial (SMJ) opera desde 2008, usa servicios de fax, correo electrónico y telefonía celular para el envío automático de notificaciones judiciales. Está integrado al sistema de notificaciones, la agenda única "Cromos"

\footnotetext{
88 Caso similar es el del Tribunal Virtual de Michigan. Véase: <http://courts.mi.gov/>

89 ELIZONDO, Macarita. "Actualización tecnológica en la administración de justicia (caso México). Revista TECSISTECATL Vol. 1 N 4. Junio 2008. México D.F.

90 Corporación excelencia en la justicia. E-Justicia, experiencias internacionales y colombianas en el uso de las TIC para el mejoramiento de la administración de justicia. Tercera versión. 2013. Véase: http://www.cej.org.co/files/Experiencias_TIC-2013.pdf. [Consultado Abril 1 de 2017].

91 México. Congreso del Estado Libre y Soberano de Nuevo León. Decreto número 160, con última reforma del 15 de abril de 2011.
} 
y el sistema de gestión de despachos judiciales, aunque la notificación se puede generar desde cualquier sistema de información del poder judicial..$^{92}$

El programa anterior demuestra lo viable de un sistema de mensajería judicial, similar al mencionado sistema de mensajería con operabilidad y seguridad adecuada que necesita Colombia para una adecuada implementación de las NE, es de apreciar que las partes involucradas en los procesos son de los principales usuarios del sistema.

\section{Conclusiones}

Las NE son el resultado de una era tecnológica en la cual se ha procurado implementar las nuevas tecnologías en la administración de justicia por su eficacia y eficiencia, y Colombia no es la excepción, por lo cual se desarrolló el tema a nivel doctrinal, legal y jurisprudencial para agilizar los diferentes procesos, y las condiciones se encuentran dadas para que nuestro país siga evolucionando en el tema de la implementación de las nuevas tecnologías en la administración de justicia.

En Colombia se han realizado diversos avances de marcada importancia en el tema, demostrando que su implementación es posible, siendo el proceso administrativo el de más avanzada y el verbal lo opuesto, aunque carecemos de sistemas de seguridad o entidades de certificación que otorgue mayor seguridad a las NE. Por lo anterior se insiste en la necesidad de un sistema de mensajería con operabilidad y seguridad adecuada dado que otorgaría mayor seguridad a las NE y permitiría proteger derechos fundamentales como el debido proceso, acceso a la justicia, seguridad jurídica, entre otros, que el Estado debe garantizar y el uso de las TIC no puede ser excusa para desconocerlos o menoscabarlos, pues son fundamentales en un estado de derecho.

Se deben encaminar los esfuerzos a tornar las NE un tipo de notificación principal, mejorando así la agilidad y eficiencia de los procesos judiciales, para lo cual se deben brindar adecuadas características de seguridad, aunque el Estado no ha tenido la debida diligencia para implementar e informar acerca de este tipo de notificación que tiene unas características y principios, especiales que bien encausados podrían derivar en el mejoramiento de la administración de justicia, agilizando los procesos.

92 Corporación excelencia en la justicia. E-Justicia, experiencias internacionales y colombianas en el uso de las TIC para el mejoramiento de la administración de justicia. Tercera versión. 2013. Véase: http://www.cej.org.co/files/Experiencias_TIC-2013.pdf. [Consultado Abril 1 de 2017]. 
Igualmente, se debe incrementar el estudio e investigación acerca del uso de las NE, pues centros de arbitramento llevan ventaja a la administración de justicia, en la implementación y desarrollo de las mismas. Las NE permiten una comunicación contigua entre la administración de justicia y las partes, aunque se deben respetar determinadas garantías, principios, reglas procesales y de seguridad mínimas para la integridad, autenticidad y confidencialidad de los documentos.

Por último se evidencia el avance por parte de diferentes países, con características socio-culturales diversas, en los cuales se han implementado las NE, junto a las nuevas tecnologías, de manera adecuada y eficaz, con una implementación paulatina, lo cual demuestra la pertinencia de realizar la misma, teniendo en cuenta que nuestra administración de justicia se encuentra colapsada.

\section{Bibliografía}

ALONSO ESPINOSA, Carles. "La información en la red y el principio de neutralidad tecnológica: la libertad de expresión y la difusión de información administrativa”. Revista derecho del Estado $N^{\circ} 22$. Junio de 2009. Bogotá D.C. Disponible en: $<$ http://revistas.uexternado.edu.co/index.php/derest/article/view /480/459>.

CARRILLO PARADA, José Rafael. 2013. "Procedimiento para las notificaciones administrativas". Revista Electrónica de la facultad de Derecho. Vol.1, Núm. 1. (2013), Universidad de Santander, UDES Cúcuta. Véase: http://service. udes.edu.co/revistas/index.php/Lex-UDES/article/P5.pdf.

CERRILLO, Agustí. "E-justicia: las tecnologías de la información y el conocimiento al servicio de la justicia iberoamericana en el siglo XXI". IDP Revista de Internet, Derecho y Política. Número 4. 2007.

Colombia. Congreso de la República. Ley 527 del 18 de 1999.

Colombia. Consejo de Estado. Sala de consulta y Servicio Civil. Oficio ํ 234. Radicado No 1989. 19 de Marzo de 2010 C.P. Enrique José Arboleda.

Colombia. Corte Constitucional. Sala plena. Sentencia de constitucionalidad N $^{\circ} 012$ del 23 de enero de 2013 M.P. González Cuervo, Mauricio. Expediente N ${ }^{\circ}$ D - 9195 .

Colombia. Corte Suprema de Justicia. Sala de Casación Penal. Sentencia del 9 de abril de 2009. M.P. Espinoza Pérez, Sigilfredo. Expediente $N^{\circ} 28909$ notificación electrónica de actos administrativos ley 1437 del 18 de enero de 2011.

Colombia. Sala Administrativa del Consejo Superior de la Judicatura. Acuerdo $\mathrm{N}^{\circ}$ PSAA06-3334 del 2 de marzo de 2006. Reglamenta los medios electrónicos e informáticos en el cumplimiento de las funciones de la administración pública. 
CORPORACIÓN EXCELENCIA EN LA JUSTICIA. E-Justicia, experiencias internacionales y colombianas en el uso de las TIC para el mejoramiento de la administración de justicia. Tercera versión. 2013. Véase: <http://www.cej.org. co/files/Experiencias_TIC-2013.pdf.>

DÍAZ GARCÍA, Alexander. "Colombia. Aspectos fundamentales del proceso, desde la óptica de las nuevas tecnologías. El documento electrónico judicial en Colombia”. Revista de derecho informático N 116. 2008 Lima.

DÍAZ GARCÍA, Alexander. Las notificaciones electrónicas judiciales en Colombia: El notario electrónico. 2008. vol.5. N ${ }^{\circ}$.2. Véase: <http://www.scielo. org.ve/scielo.php?script $=$ sci_arttext\&pid $=$ S169075152008000200008\&ln$\mathrm{g}=\mathrm{es} \& \mathrm{nrm}=\mathrm{is}>$.

FLÓREZ, Germán Darío. ”La validez jurídica de los documentos electrónicos en Colombia a partir de su evolución legislativa y jurisprudencial". Revista Verba Iuris 31. 2014. Bogotá D.C. Disponible en: <http://www.unilibre.edu.co/ verbaiuris/31/la-validez-juridica-de-los-documentos-electronicos-en-colombia-a-partir-de-su-evolucion-legislativa-y-jurisprudencial.pdf>

LILLO LOBOS, Ricardo. "El uso de las nuevas tecnologías en el sistema judicial: experiencias y precauciones". II Justicia. 2011.

MACARENO LÓPEZ, Roger Adolfo (2012) "notificación electrónica de los actos administrativos: ¿hay violación del debido proceso?”. Revista Iter Ad Veritatem 10. Universidad Santo Tomás, Tunja.

PÁJARO MORENO, Nicolás. "Las Tics al servicio del proceso". Memorias del XXXV Congreso Colombiano de Derecho Procesal. Cartagena. Septiembre de 2014.

Peña Valenzuela Daniel. De la firma manuscrita a las firmas electrónica y digital. Edición 2015. Bogotá D.C. Universidad Externado de Colombia.

QUINTERO NAVAS, Gustavo. "Contencioso administrativo y medios electrónicos: un gran paso hacia la modernización del ejercicio de la justicia administrativa". Revista de derecho, comunicaciones y nuevas tecnologías $\mathrm{N}^{\circ} 6$. Bogotá. 6 de diciembre. Universidad de los Andes. Véase: <https://derechoytics. uniandes.edu.co/components/com_revista/archivos/derechoytics/ytics103. pdf>

UMAÑA CHAUX, Andrés. "Algunos comentarios sobre el principio del equivalente funcional en la ley 527 de 1999". Revista de derecho, comunicaciones y nuevas tecnologías $\mathrm{N} 1^{\circ}$. 2005. Bogotá D.C. 